\title{
Corpus-based Stylistic Analysis of Tourism English
}

\author{
Ning Kang \\ Qingdao University of Science and Technology, Qingdao, China \\ Email: kangningkn@163.com \\ Qiaofeng $\mathrm{Yu}$ \\ Qingdao University of Science and Technology, Qingdao, China \\ Email: sunnyqf@163.com
}

\begin{abstract}
Tourism English belongs to the English for Specific Purpose (ESP) and it has its own stylistic features. This paper aims at analyzing the stylistic features of tourism English. Firstly, a large amount of authentic materials are collected from official tourism websites of Britain and the U.S., and then a corpus named Tourism English Corpus (TEC) is compiled. Freiburg-LOB Corpus of British English (FLOB) is used as the reference corpus to make comparisons. The results indicate that TEC has its own stylistic features. The average word length of TEC is a little longer than that of FLOB. As for the lexical density, there is no significant difference between the two corpora. The distribution of content words shows that content words of TEC overweigh that of FLOB with the facts that nouns are the most frequently used in both corpora and there are more nouns and adjectives but less verbs, proverbs, and pronouns in TEC. Moreover, a larger quantity of proper nouns, scenic nouns, nouns of direction, descriptive adjectives with positive meaning, general superlative adjectives, and frequent use of verbs with the meaning of visiting and enjoying are employed in TEC. Lastly, the average sentence length of TEC is shorter than that of FLOB. All these contribute to the distinctive features of tourism English. It is hoped that the research could enrich the study of tourism English and promote the translation and learning of tourism English.
\end{abstract}

Index Terms - tourism English, corpus, stylistic features

\section{INTRODUCTION}

\section{A. Purpose and Significance of the Study}

As the globalization and informationization come into full play, the chances of communication have been enhanced all over the world which also has greatly promoted the international tourism exchanges. As a result, tourism English, as an important medium of international tourism communication, is widely used in tourism industry. Tourism English has its own features in that the tourism covers large amounts of information. Moreover, eastern and western countries differ in many aspects, such as social system, historical background and geographical environment, which determine that westerners and easterners have different intentions, modes of thinking and appreciation of beauty. So people attach much importance to the study of tourism English.

Nowadays, the study of the tourism English mainly focuses on the study of genre and its functional analysis. But few studies have been conducted from the perspective of stylistic analysis. What's more, these studies are not profound enough to provide a clear framework. Therefore, it is of great significance to conduct a stylistic analysis of tourism English.

The popularization of the Internet makes tourism websites a perfect approach to gain more traveling information and attract more potential tourists. It offers a large amount of information concerning tourism. The materials to be studied in this paper are chosen from official tourism websites of Britain and the United States. At the same time, corpus has been widely employed in the study of practical problems. It has become a useful instrument for the researchers to conduct stylistic studies. Therefore, a corpus-based stylistic analysis is adopted. Generally, a corpus-based study has two functions: to provide new approaches to probe into language and to promote the theoretical thinking of language on the basis of corpus which derives from a large number of great authentic facts and statistics of language. We can observe the language patterns which we did not realize or realize little before.

This paper aims to establish a Tourism English Corpus (TEC) and conducts comparisons with FLOB-a general English Corpus. By this way, features of the tourism English will be analyzed and presented clearly, scientifically and systematically so as to change the stereotype of intuitional analysis and further promote the development of the study of tourism English.

The results of this study, on one hand, will contribute to the translation of tourism English, on the other hand, are helpful for students to learn tourism English and write this kind of articles. Furthermore, it will broaden the scope of stylistics analysis, especially stylistic analysis of tourism texts. 


\section{B. Methodology and Instruments}

The development of computer technology makes it possible to collect, label, observe and analyze linguistic data on a large scale, and it also provides people with a more macroscopical perspective to know language systematically and scientifically. Consequently, the corpus-based study is widely applied and highly popularized, and has made remarkable strides in many aspects in recent years.

Therefore, a corpus-based study is carried on in this paper to investigate the features of tourism English. The first step is to collect materials from the official tourism websites of Britain and the U.S., and then a corpus of tourism English is established after selection. The method for doing analysis is quantitative in the way of adopting some language analytical tools: Claws 4 for corpus annotation, WordSmith 3.0 and AntConc 3.2 for retrieval analysis. Besides, with the aid of FLOB (Freiburg-LOB Corpus of British English), a corpus of general English representing the language of the early 1990s, a comparison is made between language of tourism English and that of common English in order to better present the features of Tourism English.

Instruments:

Claws 4 (the Constituent Likelihood Automatic Word-tagging System) is applied in this paper for POS tagging of the corpus. Claws software was developed by the Unit for Computer Research on the English Language at Lancaster in 1980s. It has consistently achieved $96-97 \%$ accuracy.

AntConc 3.2 is free, simple, and easy to use corpus analysis software. It has several effective tools including powerful concordancers, word and keyword frequency generator, tools for cluster and lexical bundle analysis, and a word distribution, etc.

WordSmith Software designed by Mike Scott is an integrated suite for looking at how words behave in texts. It has three main functions: concordance, wordlist and key word statistics. Wordsmith 3.0 in this paper is used to calculate the Type-Token Ratio, word length and sentence length of the corpus.

The Freiburg-LOB Corpus of British (FLOB) is produced jointly with Geoffrey Leech and Nick Smith representing the language of the early 1990s. FLOB, approximate 1 million words, contains 500 texts of around 2000 words each, distributed across 15 text categories.

\section{LITERATURE REVIEW}

\section{A. Style and Stylistics}

Although style is a familiar word to us and used frequently in literary criticism and other fields, it is challenging to offer a precise definition. Crystal and Davy (1969) define that style has four aspects of meanings when investigating English style. Firstly, it may refer to some or all of the language habits (i.e. speech and writing habits) of one person. Secondly, style may refer to some or all of the language habits shared by a group of people at one time, over a period of time. Thirdly, the word "style" is given a more restricted meaning when it is used in an evaluative sense, referring to the effectiveness of a mode of expression. The last aspect of style, which is the widespread use of "style", refers to literary language.

Different situations tend to generate different varieties of a language which, in turn, show different linguistic features, so style can be seen as the various characteristic uses of language that a person or a group of persons make in various social contexts (Xu Youzhi, 1992).

Stylistics is the study of varieties of language whose properties position that language in context, and tries to establish principles capable of accounting for the particular choices made by individuals and social groups in their use of language.

According to the Longman Dictionary of Language Teaching and Applied Linguistics (Richard, 2005), stylistics is a branch of linguistics which studies the characteristics of situational-distinctive use of language, with particular reference to literary language. Xu (1992) puts forward the concept that stylistics is a discipline that studies the sum of stylistic features of the different varieties of language. $\mathrm{Hu}$ (2001) maintains that stylistics is the branch of linguistics that studies language style. It explains the relationship between the text and its context. Wright and Hope (2000) states that that stylistics studies the use of language in specific contexts and attempts to account for the characteristics that mark the language use of individuals and social group.

As mentioned above, the definitions of stylistics are all associated with style and context. Different contexts lead to different styles of writings, which become the various forms of stylistics.

\section{B. Stylistic Analysis}

Stylistic analysis, "the study of patterns formed in the process of the linguistic encoding of information, is of importance to any major research focusing upon or dependent upon the production or analysis of language" (Sedelow, 1966). According to Wang (1980), English stylistic analysis describes and investigates the language characteristics of each English style, and emphasizes that each style has its own typical ways of expression, which demand the choices of language to be equal to its purposes.

Reinhard (1995) holds the view that stylistic analysis on linguistics refers to the identification of patterns of usage in speech and writing. In other words, stylistic analysis is to analyze the use of language with the purpose of identifying some linguistic features, ranging from the general mass of linguistic feature to those which are restricted to some social 
contexts. Crystal and Davy (1969) say that stylistics analysis can be done in the lexical, grammatical, syntactic and semantic criteria.

Generally, stylistic analysis consists of qualitative analysis and quantitative analysis. Most of the previous researches apply the qualitative analysis, but it fades its color since 1960s when computational analysis came into being. Being statistic-based, immediate and practical, the corpus approach is often used to explore the subject of the quantitative stylistic analysis.

\section{Previous Study of Tourism English}

Tourism English has its own stylistic features. In general, tourism English is natural and to the point, which tends to use simple words and sentences to describe or record the concrete stuff and facts just like taking a picture. Besides, tourism English tends to use objective and concise language in a clean logical way (Ding, 2008).

Tourism English, as the name suggests, is the English language used for tourism. As "language has to serve various purposes as there are different type of occasions for using it", tourism English has its own language functions which are mainly informative function, directive function, and evocative function, or informational function, aesthetic function, descriptive function, and persuasive function ( $\mathrm{Hu}, 2001)$.

Few studies have been conducted on the stylistic features of the tourist English. Ding Dagang (2008) concluded some stylistic features of tourism: tourism English is prudent in word use, especially the key words. Loanwords, specific vocabulary, abbreviations and compound words appear frequently. Moreover, there are other characteristics: nominalization of verb phrases, the frequent usage of passive voice and present tense, the frequent employment of descriptive adjectives, superlative adjectives, imperative sentences, etc.

Sun (2009) conducts a stylistic analysis of UK's online tourist information text, in which she makes a contrast between the self-built corpus - United Kingdom's Tourist Information Texts about Tourist Attractions (UKTA) and other two corpora - JDEST (Jiao Da English for Science and Technology) and LOB (Lancaster-Oslo/Bergen Corpus), by using computational methods to conclude features of UK's online tourist information text: UKTA varies more in vocabulary than JDEST and less than LOB. As for the word length, UKTA is longer than that of LOB and shorter than that of JDEST. Large amount of nouns, adjectives and numerals are used to make the texts informative, appealing and accurate. Besides, the sentence length of UKTA is longer than that of JDEST and shorter than that of LOB. More exclamatory and imperative sentences are employed. Active sentence are preferred, while passive sentences employed are more than those of LOB and less than those of JDEST.

As mentioned above, it is only Sun Xin who makes a study on tourism English based on corpora. She collects materials only from British websites and makes analysis among self-built corpus UKTA, JDEST and LOB. Different from that, this paper establishes the corpus by collecting materials from the official websites of both Britain and the U.S., and makes contrasts between that and FLOB. FLOB is a corpus representing general English of 1990s while LOB representing general English of 1960s.

\section{PROCEDURES OF THE STUdY}

\section{A. Compiling the Tourism English Corpus}

The process of compiling the Tourism English Corpus (TEC) will be introduced in this part. There are two major steps in compiling the corpus as follows.

Sampling

In the research, samples of tourism English are chosen from official tourism websites in Britain and the U.S., such as www.enjoyengland.com, www.usatourism, www.usatourist.com, www.gohawaii.com, which are popular and accessible.These materials selected for analysis cover a wide range of landscapes: national parks, mountains, rivers, waterfalls, islands, beaches, and historical sites, etc. These natural landscapes and sceneries of humanities give an account of their history, their culture and folk customs, which illustrate different cultural backgrounds of different countries. In the process of selecting data, descriptive language is focused on but commercial and advertising information is not included. The tourism English text is saved in the format of txt as the self-built corpus.

Corpus Annotation

Corpus annotation means marking up the running words in the corpus with their part of speech (POS) tags. Therefore, the tagged corpus can be searched to perform not only the word-form but also a certain linguistic category they belong to. Tagset, a set of rules for tagging words, is the criterion for tagging every word in the corpus. Such tags are typically taken to be atomic labels attached to words, denoting the part of speech of the word, together with morphosyntactic information, e.g. they specify the word as a singular proper noun, a general comparative adjective, or an ordinal number. In the present study, we employ Claws 7 Tagset, which contains 137 tags. Claws 4 together with Claws 7 tagset is used in this paper for POS tagging. Claws software totally adopts probability statistics method, which makes the accuracy to the point of $96-97 \%$.

\section{B. Extracting Data}


In this part, quantitative analysis of tourism English will be conducted with the aid of the analysis softwareWordSmith 3.0, and AntConc 3.2. The available data are shown in tables and figures and the comparison with the corpus FLOB is made, which clearly presents the stylistic features of tourism English.

Word length

Word length constitutes as a major factor which plays a vital part in the demonstration of the characteristics of a text. As it is well known that word is the basic unit of language with the combination of form and meaning. So words with their distinguished features matter much in certain stylistic effects.

Generally speaking, the shorter and simpler the words are, the less difficult and complicated the text will be; the longer and more complex the words are, the more difficult and formal the text will be (Sun Xin, 2009).Usually, the long and difficult words are used to describe abstract, professional, or complex situations.

Word length in this paper is calculated by the letter number, which is widely adopted. Both average word length and word length are extracted by WordSmith 3.0.

Lexical Density

When it comes to lexical density, Token/Type Ratio (TTR) will be firstly introduced. The token-type ratio (TTR) is a measure of lexical density, which refers to the ratio between the types and the tokens in any given text. Token is the total number of words in a text, while type refers to the number of words which are left after the deletion of the repetition ones. Lexical density is calculated by WordSmith 3.0 and expressed as a percentage by formula:

$\mathrm{TTR}=($ NO. of tokens/NO. of types $) * 100 \%$.

Generally speaking, a higher TTR hints that a text is lexically diverse, or rich in word use, whereas, a lower TTR suggests that there are a lot of repetition of words.

However, accounting for the limitation of the English vocabulary, the number of token will increase if the text enlarges whereas the type will not grow synchronously. When the capacity of the text reaches a certain level, the augments of the type will be smaller and smaller, and the ratio between token and type cannot represent the variability of the use of words. Therefore, Standard Token/Type Ratio (STTR) has to be employed to indicate the lexical density, which is an average value of token/type ratios to be calculated in batches to length (Yang Huizhong, 2002).

Distribution of Content Words

According to Longman Dictionary of Language Teaching and Applied Linguistics (Richard, 2005), content words are words which refer to a thing, quality, state, or action and which have meanings when the words are used alone. Content words are mainly nouns, verbs, adjectives, adverbs and pronoun. This part mainly focuses on content words instead of function words, for distribution of content words could display the characteristic of a text, such as nominal preference or verbal preference. Therefore, the study of distribution of content words has practical significances in stylistic analysis of this paper.

Frequency distributions of content words of TEC and FLOB are demonstrated. When a corpus has been annotated with parts of speech, it will be easier to calculate the proportion of content words respectively, employing AntConc 3.2.

Keywords

Using WordSmith 3.0, it is possible to compare the frequencies in one wordlist against another in order to determine which words occur statistically more often. All of the words that occur more often than expected in one file when compared to another are compiled together into another list, called a keyword list.

Keywords are those words whose frequency is usually high or low in comparison with a reference corpus. Keywords analysis is useful in suggesting lexical features and further examination. This part aims to observe the top 100 keywords in detail in order to conclude the exact characteristics of tourism English.

Sentence Length

Sentence length is also one of the important factors that determine the stylistic characteristics. Basically, sentence length refers to the number of words consisting of the sentence, from the initial letter to a full-stop, a question mark or an exclamation mark. Short sentences mean simple structures which could convey meaning concisely and are easy to be understood. What's more, the information which the short sentences express is able to grasp the readers' attention swiftly. Whereas long sentences with complex structures are usually applied in more formal occasions in that they are rich in content, logical in structure, and detailed in description.

By running the WordSmith 3.0 software, the average sentence lengths in TEC and FLOB are obtained respectively.

\section{FINDINGS AND DISCUSSION}

In this part, the comparisons between TEC and FLOB are made and the extracted data are shown in tables and figures, which have clearly presented the stylistic features of tourism English.

A. Word Length

TABLE 1

AVE. WORD LENGTH (IN LETTERS) IN TEC AND FLOB

\begin{tabular}{|l|l|l|}
\hline \multicolumn{1}{|c|}{ AVE. WORD LENGTH (IN LETTERS) IN TEC AND FLOB } \\
\hline Word Length & TEC & FLOB \\
\hline Ave. Word Length (letters) & 4.77 & 4.35 \\
\hline
\end{tabular}


From Table 1 we can see that the average word length of TEC is a little longer than that of FLOB. So it suggests that the formality and difficulty of words in TEC is quite the same as in FLOB. Close to the difficulty of general English, the result is in accordance with the tourism English's feature of popularization.

$\square$ TEC $\square$ FLOB

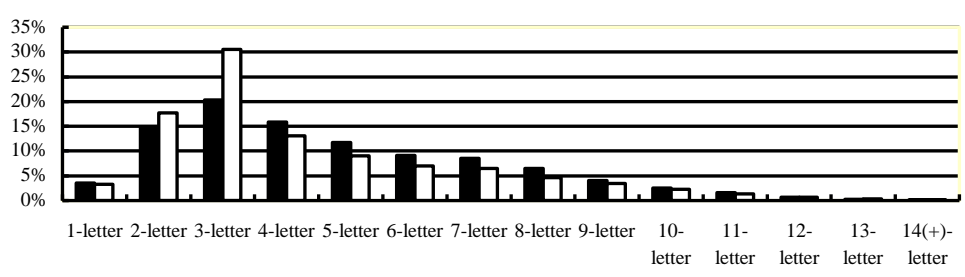

Figure 1 Word Length in TEC and FLOB

Fig. 1 gives us the details on word length in the two corpora. It is obvious that the frequent word length in these two corpora ranges from one-letter to twelve-letter. The words with thirteen or over thirteen letters are rare. The two-letter words, three-letter words, four-letter words and five-letter words account for a large percentage in both corpora. Moreover, the percentage of two-letter words and three-letter words of TEC is clearly lower than FLOB while four-letter words, five-letter words, six-letter words, seven-letter words and eight-letter words are more frequently used in TEC. The frequent use of longer words indicates that words in TEC seem a little difficult than in FLOB. It is mainly due to the fact that the tourism English is to introduce large amounts of practical information such as location, history, culture, etc. In other words, more interdisciplinary words and proper nouns occur, increasing the length and difficulty of words. In contrast, the shorter and easier words are adopted in FLOB in that they represent the general English of 1990s and need to be simple and close to daily-used ones.

B. Lexical Density

TABLE 2

COMPARISON OF STTR IN TEC AND FLOB

\begin{tabular}{|l|l|l|}
\hline TTR & TEC & FLOB \\
\hline Tokens & & \\
\hline Types & 107,981 & $1,237,424$ \\
\hline Token/Type Ratio & 10,789 & 45,089 \\
\hline Standard Token/Type Ratio & 9.99 & 3.64 \\
\hline
\end{tabular}

It can be seen clearly from the table that TTR of TEC overweighs that of FLOB. However, STTR of TEC is more or less similar to that of FLOB. That is to say, there seems little difference between the two corpora in vocabulary richness after large amounts of texts are collected.

Through analysis, the possible reasons for similar STTRs of TEC and FLOB are as follows. It is known that tourism is an interdisciplinary industry which leads to its rich vocabularies covering many aspects such as history, geography, architecture, news, religion, etc. Consequently, tourism English possesses the nature of being integrative, complicated, and interdisciplinary. At the same time, FLOB, as a corpus representing general English of 1990s, is also a mini-encyclopedia of daily life. It is no doubt that FLOB covers many fields. Both of the two corpora are representative and based on numerous text materials. As we can see, the different TTRs but similar STTR indicate that the number of tokens increases as the text enlarges whereas the types do not grow synchronously. Thus, there is an accompanying change in the ratio between token and type. Therefore, there is no difference between TEC and FLOB in lexical density according to the similar STTR.

\section{Distribution of Content Words}




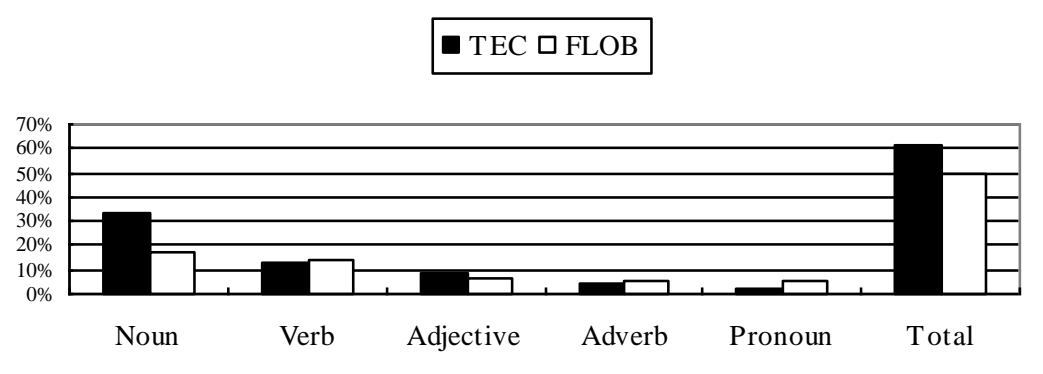

Figure 2 CONTENT Words in TEC and FLOB

From the above table, we can find that content words in TEC vary greatly from that in FLOB. The results apparently present that nouns are most frequently used in these two corpora, and verbs and adjectives also cover a high percentage. There are more nouns, adjectives but fewer verbs, adverbs and pronouns in TEC than in FLOB. Totally speaking, the percentage of content words in TEC greatly overweighs that in FLOB.

According to Ding Dagang (2008), the high percentage of nouns in TEC demonstrates the feature of tourism English. On one hand, a large number of nouns and nominal phrases contribute to a well-built, concise, clear but not complicated structure, which is easy to hold the attentions of tourists. On the other hand, tourism English tends to list concrete objectives in order to convey the actual beauty of nature. It is the faithful reappearance of the nature. Only the nouns could serve as the roles. Therefore, higher percentage of nouns but lower percentage of verbs is employed in TEC comparing to FLOB.

The purpose of an English tourism text is to attract more tourists to visit by describing the beauty of scenery. Just because of it, TEC has adopted a great number of adjectives, especially the ones with positive and cheerful meanings, to sketch the wonderland. The higher percentage of adjectives makes the famous site more romantic, fascinating and yearning. These facts better explain the higher percentage of adjectives.

\section{Keyword Analysis}

According to Ding Dagang (2008), tourism English is rigorous in word choice and deliberately chooses a large number of keywords. The characteristics of tourism English are concluded as below after comprehensive analysis of the key word list.

TEC has a higher percentage of adjectives, such as beautiful, spectacular, famous, grand, great, popular, natural etc. These adjectives are descriptive and agreeable, possessing commendatory meaning with positive emotion instead of derogatory meaning with negative emotion. Obviously, tourist text aims not only to highlight the distinguishing features of attractions but also to arouse the aesthetic interest of tourists. The colorful, vivid and fascinating introductions and descriptions tend to impress and attract more potential tourists to visit. Moreover, large amounts of adjectives could give special emphasis to the positive and enthusiasm emotion, especially the general superlative adjectives which convey strong positive emotions, such as the words largest and other adjectives with the aid of the adverb of most.

Secondly, there are many proper nouns in TEC with high keyness, such as Roman, Manhattan, California, etc. It suggests that proper nouns contribute to the features of Tourism English. Usually, when a famous place is introduced, the name of attractions, its location and history, famous people who were born there, and its beauty and magnificence would appear greatly. Therefore, a great number of proper nouns can often be seen in the tourism English, ranging from history and geography to art, architecture and religion. The keyness of proper nouns is determined by the nature of cross-disciplinary and cross-culture of tourism text.

Thirdly, scenic nouns in TEC cover a higher percentage with strong keyness, such as lake, river, mountain, island, museum, bridge, beach, etc. All these words are the targets of description that frequently occur in tourism text.

Fourthly, it is worth mentioning that there are more nouns of direction, like north, south and west, in TEC than in FLOB\&FLOWN. It is not hard to figure out the reason for their keyness. These words function as a guider to introduce the exact location of the attractions to tourists. For example, In the sentence "The third stage of Stonehenge, about 2000 BC, saw the arrival of the Sarsen Stones, which were almost certainly brought from the Marlbborough Downs near Avebury, in north Wiltshire, about 25 miles north of Stonehenge.", it has two north which gives a good illustration for the purpose of nouns of direction.

Besides, the unit of measurement, such as miles and acres, is the important keyword too. It gives assistance to the description of location and direction.

Last but not the least, among the top keywords in TEC are two verbs -visit and enjoy. The employment of these two words is in accordance with the intention of tourism English that is to arouse more and more tourists to come to visit and enjoy the beauty of nature. Such concentrated use of visit and enjoy has composed the outstanding features of TEC.

E. Sentence Length 
TABLE 3

AVERAGE SENTENCE LENGTH IN TEC AND FLOB

\begin{tabular}{|l|l|l|}
\hline \multicolumn{1}{|c|}{ Corpus } & TEC & FLOB \\
\hline Number of Sentences & 5,166 & 52,674 \\
\hline Ave. Sentence Length (letters) & 20.90 & 23.49 \\
\hline
\end{tabular}

It is apparent that the average sentence length of TEC is shorter than that of FLOB. That is to say, the sentences used in English tourism text are short containing simple structures.

As mentioned in former parts, FLOB is the reaction of all affairs of life, which covers many categories such as news, fictions, technology, entertainment, etc. To a certain extent, FLOB is the best illustration of life. Therefore, the sentences in FLOB are a little longer.

In contrast, tourism English is required to provide potential tourists with abundant and readable tourism information and attract them to visit. The intention to hold tourists' attention as soon as possible results in short and concise sentences. Moreover, TEC must be readable to tourists of different classes, nationalities and with different educational background to avoid ambiguity. These are the possible reasons for lower average sentence length in TEC.

\section{CONCLUSIONS AND LimitATIONS}

In this paper, compared with FLOB, a corpus of general English representing the language of the early 1990s, the corpus-based stylistic analysis of tourism English is carried out with the aid of annotation and statistic softwares. After a detailed exploration of tourism English, the features are concluded as follows:

(1) The average word length of TEC is a little longer than that of FLOB. Longer words seem a little more frequently used in TEC. Therefore, it can be concluded that the words used in TEC are a little more formal and difficult than in FLOB.

(2) As for the lexical density, STTR of TEC is more or less similar to that of FLOB. That is to say, there is no difference between the two corpora in vocabulary richness.

(3) The percentage of content words of TEC greatly overweighs FLOB. Nouns are most frequently used in TEC and FLOB, and verbs and adjectives cover a high percentage. There are more nouns and adjectives but fewer verbs, adverbs and pronouns in TEC than in FLOB.

(4)According to top 100 keywords, there are more proper nouns, scenic nouns, nouns of direction, the unit of measurement, descriptive adjectives with positive meaning and general superlative adjectives in TEC than in FLOB\&FLOWN. Visit and enjoy are the most frequently-used verbs in TEC.

(5) The average sentence length of TEC is shorter than that of FLOB, which means that sentences used in TEC are shorter with simpler structures.

Although we have found some stylistic features of tourism English in this paper, limitations are inevitable.

Firstly, the size of TEC is relatively small, which may affect the representativeness and accuracy of the research to some extent. Secondly, the source of the data just comes from the Internet. In the future study, the corpus should be further enlarged and the sources should also include tourist brochures, tour guidebook, etc. This paper only makes an attempt, far from sufficient. The author hopes that this paper will arouse more attention to the study of tourism English and be of great help for the learning of tourism English.

\section{REFERENCES}

[1] Bullock, A. (1978). The Fontana dictionary of modern thought. Glasgow: William Collins Sons \& Co. Ltd.

[2] Crystal, D., \& Davy, D. (1969). Investigating English Style. London: Longman Group Ltd.

[3] Ding Dagang. (2008). Features and Tourism English and Its Translation. Shanghai: Shanghai Jiao Tong University Press.

[4] Hu Zhuanglin. (2001). A Course for Linguistics. Beijing: Peking University Press.

[5] Reinhard, Kohler. (1995). Bibliography of Quantitative Linguistics. Amsterdam/Philadephia: John Benjamins Publishing Company.

[6] Richards, J.C.et al. (2005). Longman Dictionary of Language Teaching and Applied Linguistics. Beijing: Foreign Language Teaching and Research Press.

[7] Sedelow, Sally Y. (1966). A preface to computational stylistics. Netherlands: Kluwer Academic Publishers.

[8] Sun Xin. (2009). Stylistic Analysis of UK's Online Tourist Information Text. Unpublished MA thesis, Dalian Maritime University.

[9] Wang Zuoliang. (1980). Collection of Papers on English Stylistics. Beijing: Foreign Language Teaching and Research Press.

[10] Wright, L., \& Hope, J. (2000). Stylistics: A practical coursebook. Beijing: Foreign Language Teaching and Research Press.

[11] Xun Youzhi. (1992). Modern Stylistics. Kaifeng: Henan University Press.

[12] Yang Huizhong. (2002). An Introduction to Corpus Linguistics. Shanghai: Shanghai Foreign Language Education Press.

Ning Kang was born in Tangshan, China in 1972. He received his M.A. degree in foreign linguistics and applied linguistics from Dalian Maritime University, China in 2001. 
$\mathrm{He}$ is currently an associate professor in the School of Foreign Languages, Qingdao University of Science and Technology, Qingdao, China. His research interests include translation studies and corpus linguistics.

Prof. Kang is a member of Translators Association of China.

Qiaofeng Yu was born in Qingdao, China in 1977. She received her M.A. degree in foreign linguistics and applied linguistics from Donghua University, China in 2006.

She is currently a lecturer in the School of Foreign Languages, Qingdao University of Science and Technology, Qingdao, China. Her research interests include interpretation and translation studies.

Miss $\mathrm{Yu}$ is now a member of Translators Association of China. 